Results Sleep duration showed a significant inverse association with weight $(p<0.05)$ and height $(p<0.005)$ during 7 day and weekdays. A significant relationship was found between Sleep efficiency and weight, BMI, weight for age $(p<0.05)$ during weekdays and weekend days.

Conclusion In the current study, we found Sleep pattern may be an important environmental factor for obesity in school age children's. These findings are important because sleep duration is a potentially modifiable risk factor that could be important to consider in the prevention and treatment of childhood obesity.

\section{THE CHIROPRACTIC CARE OF CHILDREN IN EUROPE:RESULTS FROM A PRACTICE-BASED RESEARCH NETWORK}

doi:10.1136/archdischild-2012-302724.1672

1J Alcantara, ${ }^{2} \mathrm{~J} \mathrm{Ohm,}{ }^{2} \mathrm{~K}$ Kunz. ${ }^{1}$ Pediatric Chiropractic Research, Life Chiropractic College West, Hayward, CA; ${ }^{2}$ Research, The International Chiropractic Pediatric Association, Media, PA, USA

Background and Aims Complementary and alternative medicine (CAM) use by children is predicated upon CAM-use by their parents. Of the various practitioner-based CAM therapies for children, chiropractic is the most popular. This study was undertaken to characterize the chiropractic care of children in Europe in a practicebased research network (PBRN).

Methods This study was approved by the IRB of Life University (Marrieta, GA, USA). European chiropractors and their patients participated in a PBRN observational study to characterize the chiropractic care of children.

Results A convenience sample of 14 chiropractors (10 females; 4 males; average age $=39.36$ years; average practice experience $=$ 11.00 years) participated in this study and recruited a convenience sample of 64 parents whose child was receiving chiropractic care. Referrals patterns indicated 3 "often" received referrals from MDs while 13 indicated "often" referring patients to MDs.

Of the 64 children (34 females; 30 males; median age $=7.38$ years; complaint duration average of 1.42 years), $47 \%$ had previously or were currently attending medical care while $53 \%$ did not. Motivation for care ran the spectrum of pediatric clinical presentations from musculoskeletal (i.e., scoliosis) to non-musculoskeletal (i.e., colic, enuresis) in addition to health promotion and disease prevention. Perceived effectiveness by $72 \%$ of parents was high as monitored using a Likert Scale.

Conclusion European children attend the care of chiropractors for chronic recurrent conditions with high parental perceived effectiveness. We support further research to examine the factors for integrative pediatric care.

\section{OSTEOPATHY IN INSULIN-DEPENDANT DIABETES MELLITUS; A NEGLECTED COMPLICATION}

doi:10.1136/archdischild-2012-302724.1673

HA Bin Nakhi, N Al Zanti, Z Oabazard. Pediatrics, Adan Hospital, Kuwait, Kuwait

The main aim of management of insulin dependent diabetes (IDDM) is to prevent the acute as well as the long term complications, Recent studies suggest that IDDM in children is associated with significantly reduced bone mass density (BMD) values which may manifest as osteopenia in the growing bone. We hereby report three cases of IDDM males who were found to have low BMD and review the literature concerned with this issue.

Case 1: Eighteen years old, Kuwaiti boy known to have IDDM for last 8 years. His serum glucose is fairly well controlled with 9\% HbA1C.
Case 2: Eighteen years old, Kuwaiti boy known to have IDDM for last 5 years. His serum glucose is well controlled with $7 \%$ HbA1C.

Case 3: Nineteen years old, Kuwaiti boy known to have IDDM for last 10 years. His serum glucose is poorly controlled with $12 \%$ HbA1C. BMD was measured due to their complain of bone ache. The lumbar spine Z-scores BMD was significantly lower at the vertebrae (osteopenic range) than those of healthy population at the same age.

Conclusion \& recommendation Young males with type 1 diabetes exhibit significantly lower $\mathrm{BMD}$ values at the vertebrae which may be manifested as osteopenia in the growing bone. This may not be a late complication of type 1 diabetes and seems not to be related to the duration of the disease or to glycemic control. Therefore we recommend bone density scan for all young males with type-1diabetes to measure $\mathrm{BMD}$ and try to prevent diabetic osteopenia through therapeutic intervention.

\section{A REVIEW OF THE MEDICAL REPORTS SUBMITTED TO EDUCATION DEPARTMENT AS A PART OF THE SPECIAL NEEDS ASSESSMENT PROCESS}

doi:10.1136/archdischild-2012-302724.1674

UR Srirambhatla, H Al-Hadi. Ealing Hospital NHS Trust, Brent Community, London, UK

Background and Aims It is believed that the medical reports submitted to the Education Department are wordy and repetitive. The aims of the audit were to create clear and concise reports for the Education as a part of the special needs assessment and to test the above hypothesis.

Methods It is a retrospective qualitative analysis of the medical reports submitted to the Education department by a busy Community Paediatrics department of a District General hospital. 46 reports which were submitted to the Education from January 2011 to April 2011 were analysed through a standardised audit questionnaire.

Results Of all 46 reports, 100\% had parental views expressed, 98\% had no repetition, $86 \%$ had some documentation about the behaviour, in $85 \%$ the summary was clear and concise with appropriate recommendations, $82 \%$ had documentation about the hearing and $80 \%$ had documentation about the vision and developmental milestones. On the other hand only $56 \%$ had some documentation about the medications, $47 \%$ had a problem list, $28 \%$ had documentation of learning ability and $8 \%$ had documentation about strengths.

Conclusions The hypothesis that the medical reports are wordy and repetitive is wrong however there should be clear headings for problem list, medications, vision, hearing and developmental milestones. It was found that the existing template for the medical reports was not followed. As a result of this audit, a new template for the medical reports was developed to be used by the Community Paediatricians in an attempt to provide clear and concise reports for the Education Department.

\section{ECHOCARDIOGRAPHIC EVALUATION OF CARDIAC STRUCTURE AND FUNCTION IN OBESE EGYPTIAN ADOLESCENTS}

doi:10.1136/archdischild-2012-302724.1675

${ }^{1} \mathrm{H}$ Atwa, ${ }^{2} \mathrm{H}$ Mohamed, ${ }^{2} \mathrm{~F}$ Abbas, ${ }^{3} \mathrm{O}$ Saleh. ${ }^{1}$ Pediatric; ${ }^{2}$ Faculty of Medicine, Suez Canal University; ${ }^{3}$ Cardiology Department, Faculty of Medicine, Suez Canal University, Ismailia, Egypt

Objective To detect structural and functional changes in the left and right ventricles in obese Egyptian adolescents.

Methods Anthropometric and echocardiographic parameters, including tissue Doppler imaging, were obtained from 70 obese 
adolescents with average body mass index of 34 plus or minus 3.8 and compared with 50 age- and sex-matched controls, with a body mass index of 21.6 plus or minus 1.9. Cardiac dimensions, stroke volume, left ventricular and right ventricular systolic and diastolic functions were evaluated.

Results The obese group had a higher end-diastolic septal and posterior wall thickness and left ventricular mass index than the nonobese group. Body mass index, mid-arm and hip circumference values showed significant correlations with these echocardiographic variables. Systolic and diastolic functions of the left ventricle were normal in both groups, although stroke volume was high in the obese group. The right ventricle tissue Doppler parameters were similar in both groups. However, the S wave of the septal/lateral tricuspid valve annulus was reduced in the obese group, but not to the level reflecting systolic dysfunction. This was inversely correlated with hip, waist, and mid-arm circumference. Stepwise multiple regression analysis showed that the mid-arm and hip circumferences followed by the body mass index are significant predictors of these early cardiac abnormalities.

Conclusion Left ventricular hypertrophy is present in obese children, although both systolic and diastolic functions are normal. Tissue Doppler imaging revealed a minor, but still significant, reduction in the right ventricular systolic function.

\section{LOW DOSE OXYBUTININ IN CHILDHOOD NOCTURNAL ENURESIS}

doi:10.1136/archdischild-2012-302724.1676

M Naseri. Mashhad University of Medical Sciences, Mashhad, Iran

Objective To evaluate response to low dose oxybutynin in children with nocturnal enuresis.

Materials and Methods Forty one out of 111 neurologically normal enuretic children who referred nephrology clinic in a 3-year period (2007-2009)received low dose oxybutynin (2.5-10 mg/day depending on the age) to define response to the drug in one and 3 month periods. No response, partially and full responses were defined as decreased in bed wetting in the rate of $0-49 \%, 50-89 \%$ and $\geq 90 \%$ respectively.

Results In first month of treatment, full, partial and no responses were reported in 3 (7.3\%), 14 (34.1\%) and 24 (58.6\%) patients respectively. In non-responder patients $6(25 \%)$ and 5$) 20.8 \%$ (patients had full and partial responses in 3-month period, whereas $13(54.2 \%)$ had no response. Side effects of the drug were reported in $5(12.2 \%)$ patients. Children with non-mono symptomatic nocturnal enuresis presented a better response to the drug than those with mono symptomatic nocturnal enuresis (75\% versus $25 \%$ ). There was no significant differences between age, gender, family history of enuresis and presence of absence of daytime urinary or bowel symptoms in responder and non-responder groups ( $p>0.05$ for all).

Conclusion In the present study which is a clinical report study with no control group, there was $68.3 \%$ treatment benefit and $12 \%$ risk (side effects of the drug) with low dose oxybutynin, so it may have a role in treating nocturnal enuresis especially patients with NMNE who experience adverse effects of the drug with standard treatment.

\section{MONOSYMPTOMATIC AND NON-MONO SYMPTOMATIC NOCTURNAL ENURESIS: A CLINICAL EVALUATION}

doi:10.1136/archdischild-2012-302724.1677

'M Naseri, ${ }^{2} \mathrm{M}$ Hiradfar. 'Mashhad University of Medical Sciences; ${ }^{2}$ Pediatric Surgery, Mashhad University of Medical Sciences, Mashhad, Iran

Background Nocturnal enuresis is divided into mono-symptomatic nocturnal enuresis) MNE) and non mono-symptomatic nocturnal enuresis) NMNE. (This study was conducted to review clinical and ultrasonography findings in enuretic children, and compare organic and functional pathologies of lower urinary tract (LUT) in children with mono-MNE with those who have NMNE.

Methods 111 neurologically normal children with chief complaint of enuresis enrolled in the study including 60 boys and 51 girls, aged 5-17 years old, 43 (38.8) \% with MNE and 68 (61.2) \% with NMNE. Urine analysis, urine culture and kidney-bladder ultra sonography was done for all. Some patients underwent voiding cystoureterography (VCUG), urodynamic study (UDS), or both.

Results Patients were divided in to 3 groups: MNE, NMNE -daytime incontinence and NMNE+daytime incontinence. Constipation, encopresis and urge incontinence were significantly more frequent in patients with NMNE+daytime incontinence $(p=0.011$, $0.003,0.001$ respectively). Bladder wall thickness was the most common US findings. One patient with MNE and 9 with NMNE+ daytime incontinence had vesico-ureteral reflux (VUR) ( $p=0.016)$. Posterior urethral valve was reported in one patient with NMNE. Evidences of bladder dysfunction were noted in about half of the patients who underwent UDS, with higher prevalence in cases with NMNE+daytime urinary incontinence $(p=0.297)$. Bowel symptoms and VUR were significantly more prevalent in cases with NMNE +daytime incontinence.

Conclusion We recommend doing VCUG in enuretic children who have daytime incontinence. In addition our study revealed that symptoms suggestive of over active bladder are not good indicators for bladder dysfunction.

\section{TREATMENT OF INFANTILE COLIC WITH LACTOBACILLUS REUTERI AND THE RELATIONSHIP WITH POSTPARTUM DEPRESSION: A RANDOMIZED CONTROLLED TRIAL STUDY}

doi:10.1136/archdischild-2012-302724.1678

N Karadag, D Dilli, A Dursun, B Karagol, N Hakan, A Kundak, S Beken, A Zenciroglu, N Okumus. Dr Sami Ulus Maternity, Childrens Education and Research Hospital, Division of Neonatology, Ankara, Turkey

Background and Aim Infantile colic (IC) is a common behavioural syndrome within the first 3 months of life. We aimed to compare the effectiveness of Lactobacillus reuteri (L.reuteri) and herbal drop combined with baby massage on IC and to evaluate the relationship with postpartum depression and anxiety.

Methods Wessel Criteria were used for the diagnosis of IC in otherwise healty newborns. Patients were randomized into three groups: Group-1: L. Reuteri DSM17938 (BioGaia AB, Stockholm, Sweden), Group-2: Herbal drop (Babs, BerkoAB, İstanbul, Turkey), Group-3: Sterile water. All groups had baby massage. Infants crying episodes, feeding and stooling characteristics, growth, weight gain were noted weekly. Emotional structures of mother's behaviour were evaluated before and at 2nd month with Edinburgh postpartum depression scale (EPDS) and State Trait Anxiety Inventory (STAI) tests.

Results Seventy-four infants and their mothers completed the trial: 25 in the probiotic group, 24 in herbal drop, and 25 in placebo group. There was no difference between groups in demographic and clinical characteristics. The male rate was $62.2 \%(n=46)$. Crying times(minutes/day) at admission were similar (Group1: 348 \pm 37 , Group2: $353 \pm 30$, Group3: $346 \pm 28, p=0.67)$. A significant decrease in crying times were seen at 3.week in group1 (Group1: 35 \pm 9.8 , Group2: $300 \pm 32.2$, Group3: $188 \pm 40, p=0.001)$. The weight gain at this time was in group1 better, too $(p=0.001)$. High EPDS and STAI scores decreased significantly after treatment in group 1 at 2 month $(p=0.001)$. No adverse effects were reported in our groups.

Conclusion The emotional structure and anxiety level of mothers affects the behaviour of infants. L. reuteri, reduced the crying episodes, mothers anxiety and postpartum depression and can be used in treatment of infantile colic. 